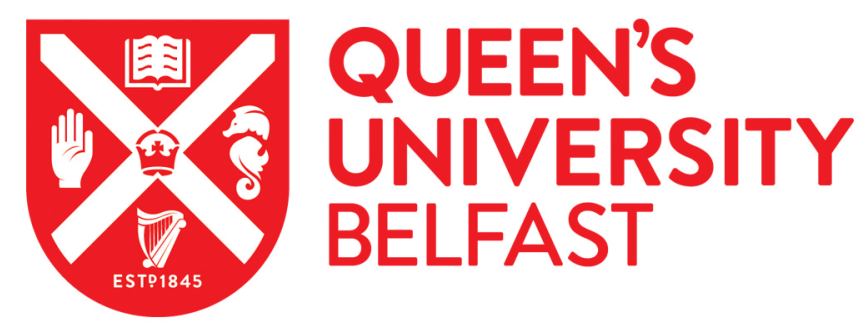

\title{
Airway clearance techniques and use of mucoactive agents for adult critically ill patients with acute respiratory failure: a qualitative study exploring UK physiotherapy practice
}

Connolly, B., Barclay, M., Blackwood, B., Bradley, J., Anand, R., Borthwick, M., Chikhani, M., Dark, P.,

Shyamsundar, M., Warburton, J., McAuley, D. F., \& O'Neill, B. (2020). Airway clearance techniques and use of mucoactive agents for adult critically ill patients with acute respiratory failure: a qualitative study exploring UK physiotherapy practice. Physiotherapy (United Kingdom), 108, 78-87.

https://doi.org/10.1016/j.physio.2020.06.003

Published in:

Physiotherapy (United Kingdom)

Document Version:

Peer reviewed version

Queen's University Belfast - Research Portal:

Link to publication record in Queen's University Belfast Research Portal

\section{Publisher rights}

Copyright 2020 Elsevier.

This manuscript is distributed under a Creative Commons Attribution-NonCommercial-NoDerivs License

(https://creativecommons.org/licenses/by-nc-nd/4.0/), which permits distribution and reproduction for non-commercial purposes, provided the author and source are cited.

\section{General rights}

Copyright for the publications made accessible via the Queen's University Belfast Research Portal is retained by the author(s) and / or other copyright owners and it is a condition of accessing these publications that users recognise and abide by the legal requirements associated with these rights.

\section{Take down policy}

The Research Portal is Queen's institutional repository that provides access to Queen's research output. Every effort has been made to ensure that content in the Research Portal does not infringe any person's rights, or applicable UK laws. If you discover content in the Research Portal that you believe breaches copyright or violates any law, please contact openaccess@qub.ac.uk. 


\section{ABSTRACT}

\section{Objectives}

3 To explore and describe current UK physiotherapy practice relating to airway clearance techniques

4 and mucoactive agents in critically ill adult patients with acute respiratory failure in the intensive care 5 unit.

\section{Design}

7 A descriptive, qualitative study using focus group interviews. Focus groups were audio-recorded,

8 independently transcribed, and data analysed thematically.

9 Participants

10 Senior, experienced physiotherapists, clinically active in critical care.

\section{Results} Fifteen physiotherapists participated in four interview sessions. Five themes emerged describing airway clearance techniques: 'Repertoire of airway clearance techniques', 'Staffing and skillset', 'Commencing respiratory physiotherapy', 'Technique selection', and 'Determining effectiveness' were themes related to airway clearance techniques. Five themes were also identified in relation to mucoactive agents: 'Use in clinical practice', 'Decision to commence', 'Selection of agent', 'Stopping mucoactive agents', and 'Determining effectiveness'. A summary of key features of standard practice was developed.

\section{Conclusions}

Standard UK physiotherapy practice of airway clearance techniques is variable, but patient-centred and targeted to individual need, with adjunctive use of mucoactive agents to enhance and optimise patient management if required. Based on this study, key features of airway clearance techniques have been summarised to help capture standard care, which could be used in future trials involving

24 ACT as part of usual care.

\section{Word count}


1. Physiotherapy practice of airway clearance techniques is patient-centred with individualised assessment to determine clinical need

2. Treatment is tailored to specific presentation, with a range of available techniques to use

3. Mucoactive agents are typically commenced to assist with management of "thick" secretions that $\underline{\text { are unable to be cleared using standard airway clearance techniques }}$

What new knowledge is added by this study?

1. Themes related to how experienced critical care physiotherapists use airway clearance techniques and mucoactive agents with critically ill patients have been generated

2. Key features characterising standard practice have been summarised

3. Practice is reflective of national guidelines

40

KEYWORDS 
Acute respiratory failure (ARF) accounts for a major proportion of admissions to the intensive care unit (ICU), with significant associated early and late mortality [1-3]. Mechanical ventilation is the cornerstone of supportive treatment [4], albeit accompanied by the risk of secretion retention secondary to altered secretion rheology and impaired mucociliary clearance $[5,6]$. Standard strategies to minimise secretion retention typically include suctioning and heated humidification [7]. Respiratory physiotherapy utilises additional airway clearance techniques (ACT) to manage focal secretion retention including manual and/or ventilator lung hyperinflation, patient positioning, and chest wall manual techniques [8-11]. These techniques may be supplemented by use of adjunctive therapies such as mucoactive agents for increased volume or tenacity of secretions [12]. Three recent studies have described detailed respiratory physiotherapy practice for mechanically ventilated patients in the ICU, all conducted in the Australia/New Zealand healthcare setting. A prospective cross-sectional point-prevalence study across $47 \mathrm{ICUs}$ and including 230 patients, reported nearly $40 \%$ of patients required additional secretion clearance techniques beyond standard suctioning, predominantly delivered by physiotherapists [10]. In a survey of senior ICU physiotherapists, $98 \%$ of respondents $(n=60 / 61$ ) identified facilitation of sputum clearance as their rationale for delivering respiratory physiotherapy [11]. High sputum load, thick secretions unable to be cleared by other modalities, and cough ineffectiveness, were the most common clinical presentations influencing duration and frequency of treatment sessions [11]. Finally, in a prospective observational study of usual care physiotherapy during acute hospitalisation, patient positioning, ventilator lung hyperinflation, and suctioning, were the most frequently performed respiratory techniques documented by physiotherapists [13].

In contrast, data describing UK respiratory physiotherapy practice for critically ill patients are limited, despite this considered an integral component of patient management $[14,15]$. Prior survey data 
79 report similarities in practice between the UK and Australia [16], albeit these data are nearly 30 years

80 old. Whilst empirically there are common professional roles and responsibilities between the

81 countries, it is uncertain that UK practice remains notably in keeping with international counterparts.

82 Furthermore, no studies of UK ICU respiratory physiotherapy have referenced use of mucoactive

83 agents as adjuncts to treatment. Empirically, Uuse of this pharmacotherapy in clinical practice is

84 common despite limited evidence for effectiveness [17, 18]. A recent UK survey of ICU-level practice

85 demonstrated the majority $(83 \%, 106 / 128$ pharmacist respondents) used mucoactive agents,

86 reported but with wide prescribing variability and minimal use of local guidelines to direct practice,

87 The most highly ranked indication for mucoactive agent use was "Thick secretions" the highest

88 indication for mucoactive agent use, and described with systemic carbocisteine and topical hypertonic

89 saline amongst the most commonly used agents_[19]-. These findings were echoed in a similar ICU-

90 clinician level survey, although predominantly ( $80 \%$ of respondents) including intensive care medicine

91 physicians [19]. The aim of our study was therefore to explore and describe current UK physiotherapy

practice relating to $\mathrm{ACT}$ and use of mucoactive agents in the management of adult critically ill patients with ARF in the ICU.

METHODS

\section{Study design}

97

This study used focus group interviews conducted between December 2018 and January 2019, led by an experienced qualitative researcher (BO'N; female, $\mathrm{PhD}$, physiotherapist). A second researcher (BC) introduced the rationale for the focus groups and intended purpose for the data acquired, and contributed additional questions at the end of the focus group to clarify or expand on content raised in the discussion. Finally, a third researcher (MB) acted as an observer. All participants had contact with the lead researcher $(\mathrm{BC})$ during the recruitment process; $B \mathrm{O}^{\prime} \mathrm{N}$ and $\mathrm{MB}$ were independent. This study was approved by King's College London Research Ethics Committee (MRA-18/19-8906), and is 
reported in line with COREQ guidelines [20]. Participants provided written informed consent prior to participation.

106

Participant selection

108

A purposive sample of participants were recruited through advertisement via social media (Twitter, including linking to the Intensive Care Society State of the Art conference), and professional physiotherapy and critical care clinical networks_(Association of Chartered Physiotherapists in

$\underline{\text { Respiratory Care; Respiratory Leaders Network) and meetings (University College London Update in }}$

112 Critical Care for Physiotherapists) [21-23]. Eligible participants were specifically-senior critical care physiotherapists (specialist grade, with at least 2 years' clinical experience), clinically active in critical care including performing ACT in the ICU. Junior rotational physiotherapists were excluded. Responders to the advertisements contacted the researchers by email and were sent a participant information sheet and details of the dates and venues of focus group sessions. All eligible participants were recruited.

\section{Data collection}

Focus groups took place in meeting rooms at an international critical care conference venue (UK) and at a London-based NHS institution. Sessions were digitally audio-recorded and independently transcribed (Accuro Transcription Solutions Limited, Cheshire, UK, www.accuro.co.uk), with additional cross-checking of transcriptions against audio-recordings for accuracy (MB). Observational field notes were made by a third researcher $(\mathrm{MB})$ to provide non-verbal contextual information to supplement the transcription. A semi-structured topic guide was used, iteratively developed and refined by the research team (Table $\underline{E} 1$ Online Supplement), with discussion reflecting ACT and mucoactive agent content. In addition, participants completed an anonymous questionnaire to provide demographic information on their clinical experience, the clinical setting of their work, and use of local guidelines or protocols regarding ACT and mucoactive agents. 
Analysis

132 Participants were given an identification code denoting their gender and-focus group session for referencing quotes. Anonymised data were analysed using thematic analysis, with themes identified at a semantic (manifest) level from direct participant phrasing [24]. Two researchers (BO'N, BC) independently analysed transcripts, examining content relating to ACT and mucoactive agent use respectively with subsequent collective review of all content. Initial themes were identified through iteratively reading of the transcripts, mapped against relevant text, and refined, condensed, or reclassified as needed. Final summary tables of themes accompanied by explanatory text and illustrative quotes were created. A third researcher $(\mathrm{MB})$ then read the transcripts and cross-checked the summary tables for accuracy and content validity, adding supplementary content and comments where necessary. Member checking, or participation validation, was conducted whereby study participants reviewed the results of the thematic analysis and the summary of key features of standard practice [25]. In addition Fthree independent senior, specialist critical care physiotherapists from different institutions reviewed the summary of key features of standard practice for additional external clinical validity. and representativeness of findings.

RESULTS

\section{Participants}

149 Twenty physiotherapists expressed interest in participating, and all were eligible. Fifteen Sixteen completed 3 focus group interviews of 2, 6, and 6 participants; and 1 individual interview. Interview 151 durations ranged from 31-46minutes. Fiveour physiotherapists were unable to attend focus groups 152 due to short notice logistical factors $(n=\underline{43})$ and health reasons $(n=1)$. Clinicians had 3-27 years critical care experience; 10 worked in general (mixed medical, surgical, trauma, severe respiratory failure services) ICUs, 4 in cardiac ICUs, and 1 in a neurological ICU; ICU bed capacity ranged 16-54 beds. Patient populations included ventilated/non-ventilated, and general medical and surgical, 

failure (including those requiring extracorporeal membrane oxygenation), and chronic respiratory disease.

\section{Thematic analysis}

Five themes each relating to both $\mathrm{ACT}$, and the use of mucoactive agents, were identified. Themes are

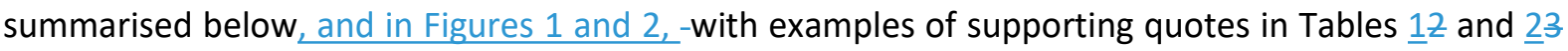
respectively. Figure 3 1-provides a summary of the key features of standard physiotherapy practice.

Themes related to airway clearance techniques

i) Repertoire of airway clearance techniques

Participants described a range of $\mathrm{ACT}$, for use in isolation or combination, and adapted these according to clinical presentation. Techniques were predominantly respiratory-based, including active cycle of breathing technique (and modified versions), autogenic drainage, manual chest wall percussion and vibration, patient positioning, manual and ventilator lung hyperinflation, recruitment manoeuvres, manual assisted cough, mechanical insufflation-exsufflation, inspiratory positive pressure breathing, flow ventilation, high frequency chest wall oscillation, and positive expiratory pressure. Humidification, use of isotonic saline nebulisers (regular or ultrasonic delivery), and suctioning were also reported. In addition, participants described utilising physical activity-based strategies including mobilisation and exercise.

\section{ii) Staffing and skillset}

178 Participants indicated that ACT practice could be influenced by physiotherapy staffing levels and 179 experience/skillset of individuals, as well as availability of senior colleagues for consultation. Weekday versus weekend delivery could also be a factor, unless units operated seven-day services, or where other staff e.g. nurses, could contribute to treatment. There was a perception that junior staff or staff 
working at weekends, for whom critical care may not be their primary speciality, may limit delivery of treatment to a narrower repertoire of ACT. In contrast, more experienced ICU physiotherapists could draw on greater experience and clinical reasoning to expand the range of ACT employed with patients. The importance of staff training was emphasised including co-working with senior critical care physiotherapists and simulation practice. In smaller ICUs maintaining skill competency and currency of multiple staff depended on regularity of access to patients requiring those particular treatments. Use of local care pathways and protocols, where available, were helpful for supporting practice, particularly for less experienced staff, while still enabling the delivery of more skilled treatments if these were needed.

\section{iii) Commencing airway clearance techniques}

Participants described varying practice around how decisions were made to commence respiratory physiotherapy with patients, but with a common emphasis on delivering individualised, clinicallyreasoned assessment and treatment. Some reported that physiotherapy staff screened all ICU patients routinely with treatment decisions based on past medical history, clinical presentation (including specific treatment pathways), and handover from the bedside nurse or multiprofessional ward round. This approach was considered valuable for providing an accurate caseload of patients requiring treatment. For others, where routine screening was not feasible or where patients were not already identified as high risk, then a referral system operated in the event of an acute episode of clinical need. This approach was sometimes considered to be less efficient if there was ultimately less urgent requirement for treatment. Inappropriate referrals, from other staff or less experienced physiotherapists, could occur where there was difficulty differentiating the clinical acuity of patients deterioration. 
209 Selection of ACT (as described in Section i) to use with patients involved assimilation of information from multiple sources - the patient's presenting condition, clinical assessment, secretion tenacity, existing type and effectiveness of secretion clearance methods, and the patient's cough strength, ability to swallow, and fatigue levels. Participants also reported that prior experience with similar patients also informed treatment selection. They described a continual cycle of clinical reasoning throughout treatment sessions, with iterative reassessment to gauge patient response. This allowed for modification of treatment depending on efficacy, supplemented by ongoing review of any updated clinical information and/or discussion with multiprofessional team members as necessary. Within specific treatment sessions, technique selection, progression, and cessation depended on the problem list and achievement of treatment goals. Participants described a range of clinical markers that guided this e.g. oxygen requirements and saturation levels, ventilator parameters, cardiovascular and respiratory stability, auscultation findings, sputum yield, and patient fatigue. Sputum quantity could have a variable impact on decision-making e.g. if high sputum load was initially problematic and then reduced, this could result in a 'scaling down' of treatment. In contrast, even if sputum yield remained high, but the patient's ability with self-clearance or routine management by nursing staff was appropriate, then physiotherapists could replace respiratory techniques for an emphasis on other components e.g. mobilisation and exercise.

\section{v) Determining effectiveness}

Outcome measures for evaluating effectiveness of ACT included subjective, e.g. more effective cough, increased ease of secretion clearance (either by clinician or independently by patient if appropriate), improved secretion tenacity, improvements in auscultation and/or palpable fremitus, and objective, e.g. peak cough flow, improvement in oxygen saturation levels, reduced $\mathrm{FiO}_{2}$ requirements, improved ventilator parameters, radiological changes on chest $\mathrm{x}$-ray. Effectiveness of treatment and subsequent decision-making about treatment were seen as interlinked. Participants reported there 
may be evidence of short-term effectiveness of ACT, where perhaps only 1-2 treatment sessions might be required before positive changes in outcome measures were seen. In other cases, longer periods of intervention might be required. Whether treatment was effective in the short- or long-term was dependent on the underlying clinical problem, and associated rationale for treatment. For example, an acute lobar collapse with sputum plugging could show signs of resolution relatively quickly with $\mathrm{ACT}$, as opposed to management of a patient with neuromuscular weakness with a low fatigue threshold, and treatment sessions may need to be adapted over time to accommodate tolerance.

\section{Themes related to mucoactive agents}

i) Use in clinical practice

Participants reported mucoactive agents were used across a wide range of patient populations, although they were not used in all ICUs. Frequently the rationale for use was to facilitate clearance of thick secretions, where existing ACT were insufficient. The definition of "thick secretions" was subjective formed by clinical judgement based on experience and individual patient presentation, with no obvious standardised approach. The notion that secretion clearance was not responsive to usual treatment was common, but the exact point at which a mucoactive agent may be required could vary between patients. Participants also described use of mucoactive agents in complex patients, where ACT may be compromised by clinical circumstances e.g. limited ability to reposition patients, or contra-indication of manual techniques. Patients with pre-existing chronic respiratory diseases could be prescribed mucoactive agents routinely if they were used as part of typical airway clearance management when clinically stable. Some participants reported that mucoactive agents were prescribed to patients according to individual clinician preference (generally the intensivist) with no specific clinical reasoning communicated. 
Participants emphasised that starting a mucoactive agent depended upon individual clinical assessment and interpretation of patients' needs, rather than a standard defined timepoint in relation to delivery of prior treatment. In many cases a hierarchical progression of treatment was evident, whereby existing ACT were optimised first, before escalating the 'intensity' of treatment to incorporate pharmacotherapy. However it was acknowledged that this treatment escalation was often non-linear and non-standardised across clinicians and across patients, due to the personalised nature of assessment and treatment in this heterogenous population. Once commenced, participants reported scheduling administration of mucoactive agents with regular ACT treatment sessions in order to maximise efficacy and benefit. Participants described a difference between those responsible for the clinical decision-making around use of a mucoactive agent with a patient, and those responsible for the actual prescribing. For the former, this could be the physiotherapist, bedside nurse or intensivist, and in many cases the decision came as a result of combined discussion (e.g. during ICU ward round) and a multi-professional approach to patient management. For the latter, prescription of the mucoactive agent was by the intensivist, or on occasion a pharmacist with prescribing rights. Physiotherapist prescribing rights (available in the UK after specific training) were considered as potentially valuable in this context.

iii) Selection of agent

277 Mucoactive agents reported included hypertonic saline, $\mathrm{N}$-acetylcysteine, and carbocisteine. Delivery could be via either nebulisation or systemic routes depending on the agent. The risk of bronchospasm as a potential side-effect of some agents (e.g. hypertonic saline) was noted, in which case concomitant use of a rescue bronchodilator was described. Participants reported that they themselves, and the wider multiprofessional teams they worked within, considered use of topical isotonic saline $(0.9 \%$, and administered either via nebulisation or instillation), to be part of usual airway clearance management. Selection of mucoactive agent prescribed tended to be the decision of the intensivist based on local availability, personal preference, or prior experience. Occasionally, institutions had locally-developed 
guidelines to support selection and use of certain mucoactive agents, albeit it was acknowledged that these guidelines were not evidence-based and predominantly informed by empirical practice.

\section{iv) Stopping mucoactive agents}

Stopping a mucoactive agent was also reportedly variable and individual according to patient progress. Symptom improvement in terms of subjective perceived secretion thickness, and objective clinical markers such as ventilator parameters, radiological changes, and respiratory values, were common reference points. In awake patients, independent ability to manage secretions efficiently and effectively was also considered. Ongoing patient monitoring and liaising with the multiprofessional team for any deterioration in patient clinical status after cessation of a mucoactive agent was also highlighted. On some occasions identifying a stopping point was empirical. Adverse events requiring a cessation of a mucoactive agent were reported as rare, and included bronchospasm, and crystallisation in the ventilator circuit tubing and/or airway.

\section{v) Determining effectiveness}

Determining effectiveness of mucoactive agents mirrored that of deciding to cease their use i.e. variable between patients, and subjective. Participants described familiarity with treating these patients meant recognition of beneficial effect was easier to detect. In essence, the agent had been effective if there was reversal of the clinical requirement that was there initially. Particular emphasis was placed on enhanced secretion clearance through reduced viscosity. Other subjective markers included that fewer ACT were needed to clear a given volume of secretions, or treatment sessions reduced in frequency and/or duration. Approaches for how clinicians judged sputum viscosity and volume were explored, with no consistent qualitative or quantitative approaches -identified; typically these were empirically assessed. Distinguishing whether improvements could be attributable to other medical treatments as part of a patient's overall management and/or resolution of the underlying 

illness could be challenging. Use of the mucoactive agent for an acute purpose or as part of longerterm management, also influenced how effectiveness was determined.

\section{DISCUSSION}

314 This is the first study to explore UK critical care physiotherapists' practice of ACT in the management of critically ill patients with ARF in the ICU, and their experience using mucoactive agents. Although practices around initiating respiratory physiotherapy varied between participants (and their respective ICUs), participants commonly described a range of ACT with selection based on individual patient assessment, clinical decision-making, and desired treatment outcome. The primary rationale for adjunctive use of mucoactive agents was presence of "thick" secretions _ secretions that are unable to be cleared using standard airway clearance techniques. Based on our results we have summarised the key features of ACT to reflect standard practice (Figure $\underline{3}_{1}$ ), which could be informative as a precis, in addition to detailed protocols, infor future interventional trials where ACT form part of usual care._This summany was reviewed and corroborated by three independent senior eritical care physiotherapists as an accurate, comprehensive, and clear, hightevel summary of clinical practice and reasoning around ACT. As a final stage of validation, this feedback suggests findings from this study are representative and generalizable to a wider population of critical care physiotherapists in 1 Us beyond those who participated in the primary focus groupsessions.

Our findings are valuable as the first to our knowledge identifying UK critical care physiotherapy around physiotherapy use of $\mathrm{ACT}_{L}$-which are consistent with those reported from other international settings both in terms of range of techniques used, and rationale $[10,11,13]$, as well as recommendations laid out in previous European guidelines [15]. Furthermore they reflect recently published updated national UK guidelines on provision of intensive care services that state "Targeted airway clearance interventions should only be considered in selected patients when clinically indicated" [14]. Our respondents indicated that use of isotonic saline was a component of usual 
airway clearance management for this patient population. This is in keeping with recent UK ICU

surveys of mucoactive agent use [26], indicating agreement across the multiprofessional team around

use of isotonic in our healthcare jurisdiction. In addition we uniquely explored use of mucoactive

agents and found these to be adjunctive to ACT to facilitate clearance of thick secretions where

existing treatments were considered insufficient. Notably, judging effectiveness of these therapies

was subjective, and future research may be valuable for developing clinically pragmatic approaches

$\underline{\text { for assessing sputum viscosity and volume. Furthermore, future-understanding ef } \text { mucoactive agent }}$

use as a component of respiratory physiotherapy this aspect of practice across international ICUs in

other healtheare jurisdictions_may be valuable for comparison to our data, and has not yet been

reported in this area $[10,11,13,27-31]$. Our study demonstrates mucoactive agent use to be an

escalation of care when existing airway clearance techniques have not been effective in these patients,

and we did not specifically explore their use in isolation, and it would be interesting to observe if this

clinical decision-making process was similar in other regions. Participants' experiences reported in

our focus groups, of the use of mucoactive agents, mirrored the results of a UK-wide survey on this aspect of practice [26]. Notably, ACT and use of mucoactive agents also both feature in minimum standards of clinical practice for physiotherapists in UK critical care; $>70 \%$ of participants (senior critical care physiotherapists) rated knowledge of the actions and implications for physiotherapy practice of mucolytics, as well as provision of a wide range of ACT such as active cycle of breathing technique, manual chest techniques, and manual hyperinflation, as essential [32], and all of which were similarly described by clinicians in the current study.

Our qualitative study included a number of methodological approaches to enhance the trustworthiness of the findings. Rigour was ensured through an a priori protocol for participant eligibility and recruitment methods, independent and external audio file transcription, and a robust data cross-checking and analysis process. We conducted focus groups to allow exploration of experiences, opinions, and beliefs, and our sample size is sufficient to have identified all themes [33]. 
362 We enrolled through discussion with multiple-senior, specialist physiotherapists, generating rich and informed data. as we anticipated these clinicians would have extensive and diverse clinical experience upon which to draw during discussions in order to generate a rich and informed dataset. However we recognise that this may be a selection bias and that other critical care physiotherapists e.g. junior rotational staff, may have opinions around experience of using ACT and mucoactive agents. As such our findings can only be considered representative of our target subgroup of physiotherapists. The focus groupthis design also enabled us to pragmatically take advantage of potential participant clinicians attending an international critical care conference, thereby maximising geographical representation. However, it could be argued that this approach also limited participation to those able to attend the conference and/or the focus group venues. We analysed data thematically at a semantic level, with no predetermined framework. This is an inductive approach whereby the verbatim descriptions provided by participants were used to characterise practice around ACT and mucoactive agents. Study participants reviewed and corroborated our thematic analysis findings and the summary of key features of standard practice [34]. They confirmed these as an accurate reflectionve of the discussions held. We took an additional, novel, step, of seeking feedback from three independent critical care physiotherapists (who met study eligibility criteria), on the summary of key features for the purposes of gauging external clinical validity of this synopsis, with feedback that this was a comprehensive, and clear, high-level summary of clinical practice and reasoning around ACT. did not show transcripts to participants for review, nor engage participants for feedback on the findings, but did provide opportunity to clarify any comments they had made and add anything further at the end of each session. We also sought independent confirmation of our summary of key findings to strengthen the robustness of this synopsis.

\section{CONCLUSION}

386 This study has shown that current UK clinical physiotherapy-practice by senior, specialist critical care physiotherapists of ACT follows general principles to deliver patient-centred individualised therapy, 
401

402

403

404

405

406

407

408

409

410

411

412

with adjunctive use of mucoactive agents to enhance and optimise patient management if required. Based on this study, key features of airway clearance techniques have been summarised to help capture standard care, which could be used in future trials involving ACT as part of usual care.

\section{ETHICAL APPROVAL}

King's College London, London, UK; Research Ethics Committee Reference: MRA-18/19-8906.

\section{FUNDING}

This research did not receive any specific grant from funding agencies in the public, commercial, or not-for-profit sectors.

\section{CONFLICT OF INTEREST}

The authors declare no conflict of interest.

\section{REFERENCES}

[1] Bellani G, Laffey JG, Pham T, Fan E, Brochard L, Esteban A, et al. Epidemiology, Patterns of Care, and Mortality for Patients With Acute Respiratory Distress Syndrome in Intensive Care Units in 50 CountriesTrends in Acute Respiratory Distress Syndrome in 50 CountriesTrends in Acute Respiratory Distress Syndrome in 50 Countries. Journal of the American Medical Association. 2016;315:788-800. [2] Prescott HC, Sjoding MW, Langa KM, Iwashyna TJ, McAuley DF. Late mortality after acute hypoxic respiratory failure. Thorax. 2018;73:618-25.

[3] Ranieri M, Rubenfeld G, Taylor Thompson B, Ferguson Niall D, Caldwell E, Fan E, et al. Acute Respiratory Distress Syndrome: The Berlin Definition. JAMA. 2012;307:2526-33.

[4] Narendra DK, Hess DR, Sessler CN, Belete HM, Guntupalli KK, Khusid F, et al. Update in Management of Severe Hypoxemic Respiratory Failure. Chest. 2017;152:867-79. 
413 [5] Konrad F, Schreiber T, Brecht-Kraus D, Georgieff M. Mucociliary Transport in ICU Patients. Chest. 1994;105:237-41.

[6] Konrad F, Schiener R, Marx T, Georgieff M. Ultrastructure and mucociliary transport of bronchial respiratory epithelium in intubated patients. Intensive Care Med. 1995;21:482-9. 2007;52:1328-47.

[8] Berney S, Haines K, Denehy L. Physiotherapy in Critical Care in Australia. Cardiopulmonary Physical Therapy Journal. 2012;23:19-25.

421 [9] Pozuelo-Carrascosa DP, Torres-Costoso A, Alvarez-Bueno C, Cavero-Redondo I, López Muñoz P, Martínez-Vizcaíno V. Multimodality respiratory physiotherapy reduces mortality but may not prevent ventilator-associated pneumonia or reduce length of stay in the intensive care unit: a systematic review. Journal of Physiotherapy. 2018;64:222-8.

[10] Ntoumenopoulos G, Hammond N, Watts NR, Thompson K, Hanlon G, Paratz JD, et al. Secretion clearance strategies in Australian and New Zealand Intensive Care Units. Aust Crit Care. 2018;31:1916.

[11] van der Lee L, Hill A-M, Patman S. A survey of clinicians regarding respiratory physiotherapy intervention for intubated and mechanically ventilated patients with community-acquired pneumonia. What is current practice in Australian ICUs? J Eval Clin Pract. 2017;23:812-20.

[12] Balsamo R, Lanata L, Egan CG. Mucoactive drugs. European Respiratory Review. 2010;19:127-33.

[13] Skinner EH, Haines KJ, Berney S, Warrillow S, Harrold M, Denehy L. Usual Care Physiotherapy During Acute Hospitalization in Subjects Admitted to the ICU: An Observational Cohort Study. Respir Care. 2015;60:1476-85.

[14] Guidelines for the Provision of Intensive Care Services, Edition 2. . Available at 19/07/2019. 
[15] Gosselink R, Bott J, Johnson M, Dean E, Nava S, Norrenberg M, et al. Physiotherapy for adult patients with critical illness: recommendations of the European Respiratory Society and European Society of Intensive Care Medicine Task Force on Physiotherapy for Critically III Patients. Intensive Care Med. 2008;34:1188-99.

442

[16] Jones AYM, Hutchinson RC, Oh TE. Chest physiotherapy practice in intensive care units in Australia, the UK and Hong Kong. Physiotherapy Theory and Practice. 1992;8:39-47.

[17] Anand R, Bradley J, Borthwick M, Connolly B, Blackwood B, Warburton J, et al. Should mucoactives be used in acute respiratory failure in the critically ill? Results from a systematic review. EP.221. Journal of the Intensive Care Society. 2018;20:Supplement 1-253.

[18] Tarrant BJ, Maitre CL, Romero L, Steward R, Button BM, Thompson BR, et al. Mucoactive agents for adults with acute lung conditions: A systematic review. Heart \& Lung: The Journal of Cardiopulmonary and Acute Care. 2019;48:141-7.

[19] Borthwick M, Shyamsundar M, Warburton J, Anand R, Bradley J, Connolly B, et al. Mucoactive agent use in adult UK Critical Care Units: a survey of health care professionals perception, pharmacists' description of practice, and point prevalence of mucoactive use in invasively mechanically ventilated patients Under Review, Peer J. 2019.

454

[20] Tong A, Craig J, Sainsbury P. Consolidated criteria for reporting qualitative research (COREQ): a

32-item checklist for interviews and focus groups. Int J Qual Health Care. 2007;19:349-57. Well-Being Research. Springer, Dordrecht.

[22] Palinkas LA, Horwitz SM, Green CA, Wisdom JP, Duan N, Hoagwood K. Purposeful Sampling for Qualitative Data Collection and Analysis in Mixed Method Implementation Research. Adm Policy Ment Health. 2015;42:533-44.

461 [23] Etikan I, Musa S, Alkassim R. Comparison of Convenience Sampling and Purposive Sampling. American Journal of Theoretical and Applied Statistics. 2016;5:1-4. 
[24] Braun V, Clarke V. Using thematic analysis in psychology. Qualitative Research in Psychology.

464

465

466

467

468

469

470

471

472

473

474

475

476

477

478

479

480

481

482

483

484

485

486

487 2006;3:77-101.

[25] Birt L, Scott S, Cavers D, Campbell C, Walter F. Member Checking:A Tool to Enhance Trustworthiness or Merely a Nod to Validation? Qual Health Res. 2016;26:1802-11.

[26] Borthwick M, Warburton J, Anand R, Bradley J, Connolly B, Blackwood B, et al. Mucoactive agent use in UK critical care units: a survey of unit-level practice. EP.133. Journal of the Intensive Care Society. 2018;20:Supplement 1-253.

[27] Bhat A, Chakravarthy K, Rao B. Chest physiotherapy techniques in neurological intensive care units of India: A survey. Indian Journal of Critical Care Medicine. 2014;18:363-8.

[28] Grammatopoulou E, Charmpas T, Strati E, Nikolaos T, Evagelodimou A, Belimpasaki V, et al. The scope of physiotherapy services provided in public ICUs in Greece: A pilot study. Physiotherapy Theory \& Practice. 2017;33:138-46.

[29] Sigera P, Tunpattu T, Jayashantha T, De Silva A, Athapattu P, Dondorp A, et al. National Profile of Physical Therapists in Critical Care Units of Sri Lanka: Lower Middle-Income Country. Phys Ther. 2016;96:933-9.

[30] Tadyanemhandu C, Manie S. Profile of patients and physiotherapy patterns in intensive care units in public hospitals in Zimbabwe: a descriptive cross-sectional study. BMC Anesthesiology. 2015;15:136.

[31] Yeole U, Chand A, Nandi B, Gawali P, Adkitte R. Physiotherapy practices in Intensive Care Units across Maharashtra. Indian Journal of Critical Care Medicine. 2015;19:669-73.

[32] Twose P, Jones U, Cornell G. Minimum standards of clinical practice for physiotherapists working in critical care settings in the United Kingdom: A modified Delphi technique. Journal of the Intensive Care Society. 2019;20:118-31.

[33] Guest G, Namey E, McKenna K. How Many Focus Groups Are Enough? Building an Evidence Base for Nonprobability Sample Sizes Field Methods. 2017;29:3-22. 
489 Care Women Int. 2007;28:888-908.

490

491

492

493

494

495

496

497

498

499

500

501

502

503

504

505

506

507

508

509

510

511

512

513

514 
516 Fable 1. Outline of focus group topic guide

Outline of focus group topic guide

Introduction about the rationale for the focus group

Discussion on what airway clearance techniques participants currently use for critically ill patients

with acute respiratory failure

Participants' rationale for determining effectiveness (or ineffectiveness) of chosen ACT in a

patient

When do participants consider using mucoactive agents with patients

Who makes the decision to use a mucoactive agent with a patient e.g. physiotherapy-led decision, medical-led decision, nurse involvement, multiprofessional team approach

What guides participants' selection of mucoactive agent and dose

How do participants' use mucoactive agents in practice

How do participants judge the effectiveness of mucoactive use, within individual treatment

sessions, and overall?

In what patients/clinical circumstances would participants not use a mucoactive agent

Have participants experienced any adverse events or complications in patients from use of

mucoactive agents

517

518

519

520

521

522

523

524 
525 Table 12. Examples of participant quotes characterising themes related to airway clearance 526 techniques

Theme

Repertoire of techniques

"...so multitudes, anything from ACBT modified, and the original version, manual techniques. We've got a Bird (intermittent positive pressure breathing) that we use sometimes on patients. We've got a cough assist that we use quite frequently with intubated and non-intubated patients. VHI, MHI and cough assist, as I've said like a manual cough assist as well" ( $\underline{P} F 1 S / 1)$

\section{Staffing and skillset}

- "I think it's almost in there's two spheres of we have a certain comfortable boundary to work within that can vary and adapt, and then there's this sphere of that's based on experience that goes a bit more than generally people feel like they're not too constrained. It's very different then when you have weekend and on-call staff." (PA41FG2)

- "...our on-call rotas are for all of our physio team barring the outpatient physios, so we really struggle to get that more technical treatments going over the weekend, and what most physios are comfortable with doing is your ACBT and your bubble pep and your manual techniques as in percussion, vibs, shaking, rolling and things like that. We really struggle with keeping things going like the hyperinflation with the cough assist and things. A lot of staff isn't comfortable with it at all...." ( $\underline{P} F 2 F G 2)$

- "we arrange our rotas so that we have seven day cover from a senior critical care physio so there's always somebody on the unit. ....." (EP4FG2)

- $\quad$ "you can deskill because you don't see them regularly enough" (PFF7FG2)

\section{Commencing airway clearance techniques}

- "... if they're on a cardiac pathway we don't see them at all and then, if they change from a respiratory point of view or a mobility point of view, then we do pick them up. And so mainly, we would pick up on handover when going around the beds every day. We are most likely to do probably a proper assessment of them and then from there determine whether they need respiratory intervention or not." ( $\underline{P} \equiv 5 F G 1)$

- "If there's no indication from airway clearance, ventilation is by minimal oxygen, no real secretion issues, then we won't actually get involved until somebody is identified or they're extubated and they're getting ready to move. But again, if up and mobile and communicating fine and there's no issues then we won't do anything, I guess our ITU caseload spans quite wide. "(

- "It's not that it's routine, it's not that there's a routine intervention expected, but there's a routine review that's expected and some of those days you find that you get to the end of your review and the outcome is endotracheal suctioning is adequate for this patient, there's no additional needs. That's the standard. That's our threshold of saying actually, we don't need physiotherapy intervention, the standard nursing practice is adequate on this patient today and that's the metaphorical threshold I guess." (APIFG2)

- "It's such a heterogeneous group and there's different things we do with airway clearance. So, if it's neuromuscular patient, yes you're going to cough assist if they're an acute airway collapse or whatever or they're post-transplant or whatever you might be more specific...." (P्F $2 F G 1)$

Technique selection

- "So, with some of the patients you might be treating them a lot acutely and then they start weaning and then you try and drop your clearance sessions, but then they start to go into weaning so then you reintroduce it, like again. But that's probably no more than your 
neuromuscular patients sometimes. That I will look back and say oh, they're failing to wean as well now and secretion clearance is the issue." (PÆ3FG1)

- "you're just looking at more respiratory function as a whole to improve oxygenation side of things, rather than just purely focussing on the sputum clearance." (PAA1FG3)

- 'Yes, you've got a problem list in your head and that's what you're trying to solve or at least improve. And then in your head you have kind of got an endpoint already for what your goals are in a way, and then throughout you're constantly reassessing to make sure the patient is stable throughout and they're tolerating the treatment okay, adapting your treatment accordingly " (FP2FG1)

"It depends on each patient" (ㅍ6FG2)

\section{Determining effectiveness}

- "Sometimes getting a big glob of phlegm does mean you're effective because it can be that that glob of phlegm is the one that's causing the problem". (PF6FG2)

- "About half an hour later they will pick up their stats and are an awful lot better...Someone's chest $x$-rays may change dramatically and another person's may not." (FPGFG2)

- "I think progress of weaning as well. So, with some of the patients you might be treating them a lot acutely and then they start weaning and then you try and drop your clearance sessions, but then they start to weaning so then you might reintroduce it, like again. But that's probably more than your neuromuscular patients sometimes. That I will look back and say oh, they're failing to wean as well now and secretion clearance is the issue." (PF $3 F G 1$ )

- "No, I think there's definitely long-term impact in there somewhere. It's incredibly hard to isolate and define, but I definitely think what we do has got more than just a short-term impact. And I think, if you can clear someone's chest to give them a little bit of rest overnight and actually, they feel a lot brighter and they're able to do a lot more the next day, I think that's something." (P正FG2) 
540 Table 23. Examples of participant quotes characterising themes related to mucoactive agents

Theme

Use in clinical practice

- "We tend not to use mucolytics to be honest. We only use it if they are already on existing treatment." (P्PF5FG2)

- "Ours is dependent on which ITU consultant is on...you come in and everyone's on NAC nebulisers and then you come in the next day and they're all off it again" (P巨FGG2)

- "severe respiratory failure patients so their secretions are really tenacious... we feel that clearance would be more effectives if we gave a mucolytic agent prior to physiotherapy we would recommend that or regular mucolytics throughout the day so that when physio comes they've had a good few doses of it" (

- '...it's definitely an individualised clinical assessment based on patient's needs, we don't have a timeframe as in after three or four days, if we are still requiring VYI or something we definitely add in a mucolytic or mucolytic active agent. You could add in day one if that was what you felt through your assessment." ( $\underline{P \models 1 S I 1)}$

\section{Decision to commence}

- "...you've probably tried your airway clearance techniques first obviously escalating them as need be...if you're clearing small amounts, that are really thick, tenacious, difficult to clear up the suction catheter...if you're trying everything at that point and you can't actually, this isn't helping then you definitely need mucolytics then as well" (

- "Thick secretions which are difficult to clear with the airway clearance techniques we've discussed. Because as well we said about upping the frequency (of treatment) but there's always a limit to what almost a patient can tolerate but also what resources you have to keep treating. That has to come into it to some extent..." ( $\underline{P} F 2 F G 1)$

- "We instil it a lot, a huge amount actually... with no issues... we don't tend to advocate the 0.9 with saline nebs just when they are intubated and ventilated because they are all on heated humidification so we didn't feel it added that much.... We don't really have a guideline on how much because when they did bronchs here they will put so much down that the Consultants don't have a problem with us doing that at all and the nurses will do it as well. I think there is quite an ethos of that and I do think it helps with secretion clearance absolutely...provides a medium for it to move and it might trigger a cough reflex so that's kind of why we are doing it. But the long term it doesn't have a breakdown like NAC would do" ( $\underline{P} \equiv 1 S I 1)$

- "It would be discussed on the ward round. Medics, nurse, pharmacy, physio would be on the ward round every day, so you would have a role but it would be a team decision." (P्PFFG2)

- "Usually we're the ones who will know...rather than a discussion we're usually the ones leading, identifying the need... I don't think our interventions are escalated in a linear fashion, it's a lot messier than that..." (PAM1FG2)

\section{Selection of agent}

- "So we have a guideline for the unit (for using mucolytics)... based on a literature review and evidence review but obviously the evidence basis is quite loose at the moment" (퓌SI1)

- "It depends what it is so like carbocisteine will probably be like oral TDS and so that just happens in the background. NAC nebs, I don't think necessarily, I think they get prescribed regularly normally and I would just treat whenever. Whereas hypertonic saline, I would more likely plan a treatment around, I guess." ( $\underline{P} \mp 4 F G 2)$

- "I feel like we're quite systematic about, or I am as a clinician quite systematic about my use of airway clearance techniques and completely non-systematic about whether or not mucolytics are added in." ( $\underline{P} \mp 4 F G 2)$

\section{Stopping mucoactive agents}


- " "If the patient gets better it might stop." ( $\underline{P} F 3 F G 1)$

- "Less mucus production, inflammatory markers going down, chest x-ray changes improving, gases. If there's a general trend towards improvement and there's no chronic underlying condition that that needs them on it full time anyway." ( $\underline{P} F 2 F G 1)$

- "So it's a joint decision." ( $\underline{P} F 5 F G 1)$

- "I think obviously we're interacting with patients from a respiratory perspective probably more so than the medical team....they rely on our feedback..." ( $\underline{P E T F G 1)}$

"...sputum viscosity, but it's very subjective." ( $\underline{P} \mp 7 F G 2)$

\section{Determining effectiveness}

- '...the sputum viscosity, but it's very subjective. I don't think there is a measure I look at and I would think this is now the viscosity of my sputum but it's still the speed it comes up the catheter and how easy it's clearing, and when the nurses start to clear it and all the other objective measures, work of breathing, FiO2 etc." (PEFFG2)

- "It seems to be quite subjective in terms of whether you're getting more secretions to the volume, but we are not technically measuring volume we just are sort of looking at suction tubing and as I've said it's very individual, I suppose" ( $\underline{P} \equiv 1 S / 1$ )

- "If you have treated that patient a lot I think you'd have a much better idea about when you thought you were clearing more. Whether the secretions were looser than previously...." (P्PE1SI1) 
557

FIGURES

$558 \quad$ Figure 1. Summary of themes reflecting airway clearance techniques

559

$560 \quad$ Figure 2. Summary of themes reflecting mucoactive agent use

561

562 Figure 31. Summary of the key features of airway clearance techniques reflecting standard practice 563

564 